\title{
CRECIMIENTO DE CAFÉ (Coffea arabica L.) DURANTE LA ETAPA DE ALMÁCIGO EN RESPUESTA A LA SALINIDAD GENERADA POR FERTILIZANTES
}

\section{GROWTH OF COFFEE (Coffea arabica L.) DURING NURSERY'S STAGE IN RESPONSE TO THE SALINITY GENERATED BY FERTILIZERS}

\author{
Siavosh Sadeghian Khalajabadi'; Raúl Darío Zapata H. ${ }^{2}$
}

Fecha de recepción: Octubre 10 de 2014 Fecha de aceptación: Noviembre 14 de 2015

\begin{abstract}
RESUMEN
La salinidad del suelo puede afectar negativamente el crecimiento de las plantas, e incluso causar su muerte. Esta investigación tuvo como propósito, determinar el efecto de la salinidad generada por fertilizantes en el crecimiento de café Coffea arabica L. durante la etapa de almácigo. Se recolectaron muestras de suelo en dos Estaciones Experimentales (EE) de Cenicafé (Naranjal en Caldas y Timbío en Cauca) y una finca cafetera (FC) en Jamundí (Valle del Cauca). Bajo el diseño completamente aleatorio se evaluó para cada suelo la respuesta de café variedad Caturra a 27 tratamientos $\left(\right.$ factorial $\left.3^{3}\right)$, resultantes de tres dosis de calcio-Ca, de magnesio-Mg y de potasio-K $\left(0,1\right.$ y $3 \mathrm{~g} \mathrm{dm}^{-3}$ de suelo), empleando como fuentes sulfatos. La conductividad eléctrica (C.E.), como indicador de la salinidad, se incrementó en conformidad a las dosis, con valores más altos para el Mg, seguido por $\mathrm{K}$ y Ca, resultado que se relacionó principalmente con el índice de salinidad de los fertilizantes y su solubilidad. Las diferencias de la C.E. entre las localidades (EE Naranjal>EE Timbío>FC Jamundí) se atribuyeron a la capacidad de éstos para retener la humedad. Conforme al aumento
\end{abstract}

1 Ph.D. Centro Nacional de Investigaciones de Café-CENICAFÉ, Universidad Nacional de Colombia-sede Medellín, Medellín, Colombia.Siavosh.Sadeghian@cafedecolombia.com,rdzapata@unal.edu.co

2 Docente.Ph.D. Universidad Nacional de Colombia. Medellín.Colombia.rdzapata@unal.edu.co 
de la salinidad disminuyó el peso de las plantas, hasta causar síntomas de toxicidad y posterior muerte. El nivel crítico de la C.E., calculado para el 90\% del crecimiento relativo, correspondió en promedio a $1,1 \mathrm{dS} \mathrm{m}^{-1}$.

Palabras clave: Conductividad eléctrica, toxicidad, fuentes fertilizantes, retención de humedad.

\begin{abstract}
Soil salinity can negatively affect plant growth and even cause their death. This research aimed to determine the effect of the salinity generated by fertilizers on coffee (Coffea Arabica L.) growth during the nursery stage. Soil samples were collected at two Experimental Stations (ES) of Cenicafé (Naranjal in the department of Caldas and Timbío in the department of Cauca) and at a coffee farm in Jamundí (Valle del Cauca). For each soil, the response of coffee Caturra variety was evaluated through a complete random design and 27 treatments (factorial $3^{3}$ ), resulting from three doses of calcium-Ca, magnesium- $\mathrm{Mg}$, and potassium-K $\left(0,1\right.$, and $3 \mathrm{~g} \mathrm{dm}^{-3}$ of soil), using sulfates sources. The electrical conductivity (E.C.), as an indicator of salinity, increased in accordance with the applied doses, with higher values for $\mathrm{Mg}$, followed by $\mathrm{K}$ and $\mathrm{Ca}$. This result was primarily related to the salinity index of the fertilizers and their solubility. The differences in E.C. between localities (EE Naranjal>EE Timbío>FC Jamundí) were attributed to their humidity retention capacity. As salinity increased, plant weight decreased, until causing toxicity symptoms and subsequent death. The critical level of E.C., calculated for $90 \%$ of the relative growth, corresponded to an average of $1.1 \mathrm{dS} \mathrm{m}^{-1}$.
\end{abstract}

Keywords: Electrical conductivity, toxicity, fertilizer sources, humidity retention.

\section{INTRODUCCIÓN}

La obtención de almácigos vigorosos de café (Coffea arabica L.) es uno de los pilares fundamentales en el establecimiento de los cultivos que pueden permanecer por más de 15 años en el campo. Entre los factores de éxito para lograr este objetivo está la adecuada nutrición de las plantas, la cual depende entre otros aspectos, de la selección apropiada de la dosis y la fuente de cada elemento. El exceso en la cantidad de un fertilizante puede generar desbalances nutricionales, con consecuencias negativas en el crecimiento; en casos severos se produce toxicidad e incluso la muerte de la planta. Son ejemplos de esta situación el empleo de abonos orgánicos que no están suficientemente descompuestos (Salamanca y Sadeghian, 2008; Ávila et al., 2010) y el sobre-encalamiento (Sadeghian, 2013).

Un caso particular ocurre con la aplicación de cantidades elevadas de fertilizantes, práctica que incrementa la salinidad del suelo y por ende, la presión osmótica del agua en el medio donde crecen las raíces de las plantas hasta niveles intolerantes (Faria et al., 2009). Una alta presión osmótica en la solución del suelo reduce la energía libre del agua, afectando de manera negativa la absorción de ésta y algunos nutrientes (Marschner, 1995). La magnitud del estrés que genera la salinidad en la planta depende de factores como el cultivar, 
el tipo, intensidad y duración de la salinidad, la luminosidad, la humedad del suelo y del aire, y de la etapa del desarrollo de la planta (Cramer et al., 1994).

En Colombia rara vez se presentan problemas de salinidad en el cultivo de café en el campo, situación diferente a lo que ocurre en países como Brasil, donde se realizan prácticas de riego con aguas salinas o fertirriego. Faria et al. (2009), Figueirêdo et al. (2006), Karasawa et al. (2003) y Nazário et al. (2010), encontraron que la salinidad puede afectar las diferentes variables biológicas de café, entre ellas área foliar, altura de la planta, acumulación de materia seca de la parte aérea y radical, potencial de agua en las hojas y actividad de nitrato reductasa y en casos extremos causar la muerte de las plantas en las etapas iníciales de crecimiento (Faria et al., 2009; Figueirêdo et al., 2006).

Dado que a nivel nacional no hay información acerca del tema, el siguiente experimento tuvo como propósito determinar el efecto de la salinidad del suelo, causada por fertilizantes, en el crecimiento de café durante la etapa de almácigo.

\section{MATERIALES Y MÉTODOS}

La investigación se desarrolló bajo condiciones de casa de malla en el Centro Nacional de Investigaciones de Café (CENICAFÉ), ubicado en el municipio de Chinchiná, departamento de Caldas, a $5^{\circ} 1^{\prime}$ latitud norte y $75^{\circ} 35^{\prime}$ longitud oeste, a $1310 \mathrm{msnm}$, temperatura promedia de $21,7^{\circ} \mathrm{C}$ y precipitación anual de $3000 \mathrm{~mm}$.

Se recolectó aproximadamente $1 \mathrm{~m}^{3}$ de suelo a $25 \mathrm{~cm}$ de profundidad en dos Estaciones Experimentales (EE) de CENICAFÉ y una finca cafetera (FC) (Tabla 1). Al suelo recolectado en cada una de las localidades se aplicaron 27 tratamientos, resultantes de tres dosis de calcioCa $\left(0,1\right.$ y $3 \mathrm{~g} \mathrm{dm}^{-3}$ de suelo) en combinación con tres dosis de magnesio- $\mathrm{Mg}\left(0,1\right.$ y $3 \mathrm{~g} \mathrm{dm}^{-3}$ de suelo) y tres dosis de potasio-K $\left(0,1\right.$ y $3 \mathrm{~g} \mathrm{dm}^{-3}$ de suelo), empleando como fuentes sulfato de calcio- $\mathrm{CaSO}_{4} \cdot 2 \mathrm{H}_{2} \mathrm{O}(28 \%$ de $\mathrm{CaO}$ y $18 \%$ de $\mathrm{S})$, sulfato de magnesio- $\mathrm{MgSO}_{4} \cdot 7 \mathrm{H}_{2} \mathrm{O}(16,4 \%$ de $\mathrm{MgO}$ y $13 \%$ de S) y sulfato de potasio- $\mathrm{K}_{2} \mathrm{SO}_{4}$ (50\% de $\mathrm{K}_{2} \mathrm{O}$ y $18 \%$ de $\mathrm{S}$ ). Una vez incorporados los fertilizantes al suelo en cada tratamiento, se procedió a llenar con la mezcla resultante bolsas plásticas de polietileno color negro de aproximadamente $2 \mathrm{dm}^{3}(17 \mathrm{~cm}$ de alto y $23 \mathrm{~cm}$ de ancho). Posteriormente los suelos se humedecieron hasta alcanzar su máxima capacidad de retención de agua y se incubaron por 20 días; una vez transcurrido este tiempo, se trasplantó en cada bolsa una plántula de café variedad Caturra, cuyas semillas previamente habían germinado en arena de río lavada y desinfestada.

A partir de ésta fecha, las plantas recibieron un manejo agronómico, siguiendo las recomendaciones de la Federación Nacional de Cafeteros de Colombia para esta etapa fenológica del cultivo, concerniente a control de arvenses, riego, sombra y manejo fitosanitario. Dos meses después del trasplante se aplicaron $2 \mathrm{~g}$ de fósforo $\left(\mathrm{P}_{2} \mathrm{O}_{5}\right)$ en forma de fosfato diamónico-DAP (18\% de N y $46 \%$ de $\mathrm{P}_{2} \mathrm{O}_{5}$ ), de acuerdo a lo recomendado para la fase de almácigos (Sadeghian, 2013).

El diseño experimental fue completamente al azar en arreglo factorial de $3^{3}$ ( 3 dosis de $\mathrm{Ca}$ x 3 dosis de $\mathrm{Mg}$ x 3 dosis de $\mathrm{K}$ ), y cada tratamiento contó con 10 repeticiones por lo tanto, por cada localidad se tuvieron 270 unidades experimentales.

Seis meses después de la siembra, tiempo correspondiente a la etapa de almácigo, definido para 
bolsas de $2 \mathrm{dm}^{-3}$, se tomaron muestras de suelo en cuatro de las diez repeticiones, con el fin de analizar la conductividad eléctrica (C.E.) como indicador de la salinidad. Posteriormente, se separaron las raíces del suelo mediante la aplicación de agua y se secaron las plantas en estufa durante 72 horas a $65^{\circ} \mathrm{C}$.
Para cada localidad se hizo un análisis de varianza con un nivel de confianza del $95 \%$; así mismo, mediante el uso de regresiones se seleccionaron modelos para explicar el efecto de las dosis suministradas en las variables de respuesta (conductividad eléctrica y materia seca de las plantas).

Tabla 1. Procedencia, unidad cartográfica, clasificación taxonómica, propiedades químicas y físicas de las muestras de suelo empleadas

\begin{tabular}{|c|c|c|c|}
\hline & EE Naranjal & EE Timbío & FC Jamundí \\
\hline & \multicolumn{3}{|c|}{ Procedencia } \\
\hline Municipio & Chinchiná & Timbío & Jamundí \\
\hline Departamento & Caldas & Cauca & Valle del Cauca \\
\hline \multicolumn{4}{|c|}{ Unidad cartográfica y clase taxonómica } \\
\hline Unidad cartográfica & Chinchiná & Timbío & Doscientos \\
\hline Clase taxonómica & $\begin{array}{c}\text { Acrudoxic } \\
\text { Melanudands }\end{array}$ & Hydric Fulvudands & Typic Dystropepts \\
\hline \multicolumn{4}{|c|}{ Propiedades químicas } \\
\hline$\overline{\mathrm{pH}}$ & 4,80 & 4,90 & 5,30 \\
\hline $\mathrm{CO}(\%)$ & 7,83 & 14,04 & 4,06 \\
\hline $\mathrm{N}(\%)$ & 0,51 & 0,75 & 0,30 \\
\hline $\mathrm{P}\left(\mathrm{mg} \mathrm{kg}{ }^{-1}\right)$ & 21,00 & 3,00 & 9,00 \\
\hline $\left.\mathrm{S}(\mathrm{mg} \mathrm{kg})^{-1}\right)$ & 23,30 & 3,90 & 11,80 \\
\hline $\mathrm{Ca}\left(\mathrm{cmol}_{\mathrm{c}} \mathrm{kg}^{-1}\right)$ & 1,00 & 3,80 & 7,70 \\
\hline $\mathrm{Mg}\left(\mathrm{cmol}_{\mathrm{c}} \mathrm{kg}^{-1}\right)$ & 0,50 & 1,31 & 4,50 \\
\hline $\mathrm{K}\left(\mathrm{cmol}_{\mathrm{c}} \mathrm{kg}^{-1}\right)$ & 1,56 & 1,72 & 1,91 \\
\hline $\mathrm{Na}\left(\mathrm{cmol}_{\mathrm{c}} \mathrm{kg}^{-1}\right)$ & 0,04 & 0,02 & 0,04 \\
\hline $\mathrm{Al}\left(\mathrm{cmol}_{\mathrm{c}}^{\mathrm{c}} \mathrm{kg}^{-1}\right)$ & 1,10 & 0,90 & 0,30 \\
\hline $\mathrm{CIC}\left(\mathrm{cmol}_{\mathrm{c}} \mathrm{kg}^{-1}\right)$ & 20,0 & 28,0 & 23,0 \\
\hline $\mathrm{Fe}\left(\mathrm{mg} \mathrm{kg}^{-1}\right)$ & 191 & 315 & 279 \\
\hline $\operatorname{Mn}\left(\mathrm{mg} \mathrm{kg}^{-1}\right)$ & 18 & 35 & 83 \\
\hline $\mathrm{Zn}\left(\mathrm{mg} \mathrm{kg}^{-1}\right)$ & 4,0 & 9,0 & 9,0 \\
\hline $\mathrm{Cu}\left(\mathrm{mg} \mathrm{kg}^{-1}\right)$ & 7,0 & 3,0 & 20,0 \\
\hline $\mathrm{B}\left(\mathrm{mg} \mathrm{kg}^{-1}\right)$ & 0,46 & 0,25 & 0,54 \\
\hline \multicolumn{4}{|c|}{ Propiedades físicas } \\
\hline$\overline{\operatorname{Ar}(\%)}$ & 23,0 & 27,0 & 41,0 \\
\hline $\mathrm{L}(\%)$ & 29,0 & 32,0 & 25,0 \\
\hline $\mathrm{A}(\%)$ & 49,0 & 41,0 & 35,0 \\
\hline Textura & $\mathrm{F}$ & Far & $\mathrm{Ar}$ \\
\hline $\mathrm{DA}\left(\mathrm{g} \mathrm{cm}^{-3}\right)$ & 0,76 & 0,54 & 0,96 \\
\hline Porosidad total (\%) & 67,76 & 73,46 & 63,20 \\
\hline Macroporos (\%) & 20,84 & 29,30 & 20,25 \\
\hline Mesoporos (\%) & 18,57 & 13,26 & 5,99 \\
\hline Microporos (\%) & 28,35 & 30,91 & 36,96 \\
\hline
\end{tabular}

EE: Estación Experimental. FC: Finca Cafetera. DA: Densidad aparente. 


\section{RESULTADOS Y DISCUSIÓN}

\section{Efecto de los tratamientos en la salinidad del suelo}

En el tratamiento testigo sin el aporte de $\mathrm{Ca}, \mathrm{Mg}$ ni K, pero con la aplicación de DAP, la conductividad eléctrica (C.E.) para los suelos de la EE Naranjal, EE Timbío y FC Jamundí fue de $1,5 \mathrm{dS} \mathrm{m}^{-1}$, $1,0 \mathrm{dS} \mathrm{m}^{-1}$ y $0,9 \mathrm{dS} \mathrm{m}^{-1}$, respectivamente. Según los rangos generales establecidos para Colombia por el ICA (1992), estos valores son relativamente bajos y se consideran normales para la zona cafetera del país, en donde las condiciones de clima (principalmente la precipitación), relieve y el origen de los suelos, no favorecen la salinidad.

Conforme al aumento en las cantidades de los fertilizantes, se incrementó la C.E. (Tabla 2). Cuando se aplicó la dosis más alta de Ca (3 g $\left.\mathrm{dm}^{-3}\right)$, sin incluir $\mathrm{Mg}$ ni $\mathrm{K}$, se lograron conductividades eléctricas entre 1,6 y 2,07 dS $\mathrm{m}^{-1}$, al suministrar $\mathrm{K}$ en las mismas cantidades, sin el aporte de los otros dos elementos, los valores alcanzados estuvieron entre 1,9 y 2,8 dS $\mathrm{m}^{-1}, \mathrm{y}$ al proporcionar $3 \mathrm{~g} \mathrm{dm}^{-3}$ de $\mathrm{Mg}$ en ausencia de Ca y K, se consiguieron conductividades desde 2,4 hasta 5,1 dS m-1. Estas diferencias se relacionan directamente con las propiedades de las fuentes empleadas; al respecto, el sulfato de $\mathrm{Ca}$ presenta un bajo índice de salinidad $(8,1)$ con respecto a los sulfatos de $\mathrm{Mg}$ y de K (46,1 y 44,0, respectivamente), tomando como referente al $\mathrm{NaNO}_{3}=100$ (Guerrero, 2004). Adicionalmente, las cantidades suministradas de sulfato de $\mathrm{Mg}$ fueron mayores a las otras dos fuentes en cualquiera de las dosis, como consecuencia de su menor concentración $(16,4 \%$ de $\mathrm{MgO}$, frente a $28 \%$ de $\mathrm{CaO}$ y $50 \%$ de $\mathrm{K}_{2} \mathrm{O}$ ). Otro aspecto a tener en cuenta tiene que ver con la dificultad que tienen las fuentes empleadas para disolverse; consecuentemente, la solubilidad del sulfato de

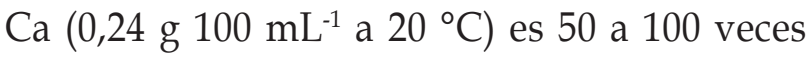

menor que los sulfatos de $\mathrm{K}$ y $\mathrm{Mg}(12$ y $26 \mathrm{~g}$ $100 \mathrm{~mL}^{-1}$, respectivamente) (Guerrero, 2004). A lo anterior se adiciona la facilidad que presenta el $\mathrm{Ca}^{2+}$ para precipitarse en presencia del ion sulfato y así permanecer en la matriz del suelo (Lindsay, 2001).

De acuerdo con los criterios establecidos por Richards (1954) en el Manual 60 de USDA, y adoptado mundialmente hasta el día de hoy, un suelo se clasifica como salino cuando la C.E. de éste sea igual o mayor a 4,0 dS $\mathrm{m}^{-1}$, valor crítico para el cual se espera una reducción del $50 \%$ en la producción de la mayoría de los cultivos. García (2010), aclara que un suelo empieza a presentar problemas de sales cuando su C.E. es superior a $2 \mathrm{dS} \mathrm{cm} \mathrm{cm}^{-1}$ y añade que el límite de la clasificación como salino depende de la especie. Por lo tanto y basándose en los resultados obtenidos, se puede afirmar que la aplicación de los tres elementos, en especial el Mg y el K, generó salinidad en los tres suelos conforme a las dosis suministradas, hasta alcanzar niveles que superan los valores críticos reportados.

Al considerar los promedios obtenidos para cada dosis, se corrobora la menor influencia que tuvo el suministro de Ca en la salinidad de las tres localidades (Fig. 1) conservando en todos los casos el siguiente orden: EE Naranjal > EE Timbío > FC Jamundí. Las pendientes de las regresiones indican además que los incrementos debidos al Ca fueron bajos y similares para los tres suelos (pendientes entre 0,1213 y 0,1427); en contraste, mediante la aplicación de $\mathrm{Mg}$ se registraron los mayores aumentos en todos los suelos, en especial para EE Naranjal (pendiente de la regresión $=0,8410$ ).

Las diferencias detectadas en la C.E. entre los tres suelos, están relacionadas principalmente con la habilidad de éstos para retener las sales o permitir que drenen una vez que estén disueltas. Entre las 
propiedades que condicionan dicha habilidad, está la porosidad y la respectiva distribución relativa del tamaño de los poros (Amézquita, 2001). En los suelos de Naranjal y Jamundí el volumen de los espacios encargados del drenaje (macroporos), fue relativamente similar (Tab. 1), en tanto que la fracción para retener el agua disponible para las plantas, representada por los mesoporos, fue menor en el suelo de FC Jamundí $(5,99 \%)$, con respecto a EE Timbío $(13,26 \%)$ y EE Naranjal (18,57\%), diferencia que contribuyó a la salida del agua hacia el especio exterior de las bolsas mediante los constantes riegos, reduciendo así la conductividad eléctrica y la concentración de los sulfatos como aniones acompañantes de las bases suministradas a través de los fertilizantes.

Tabla 2. Valores promedio de la conductividad eléctrica $\left(\mathrm{dS} \mathrm{m}^{-1}\right)$ y la respectiva desviación estándar (D.E.), obtenidos para los suelos de tres localidades de la zona cafetera en respuesta a las dosis de $\mathrm{Ca}, \mathrm{Mg}$ y K

\begin{tabular}{|c|c|c|c|c|c|c|c|c|}
\hline \multicolumn{3}{|c|}{ Dosis $\left(\mathrm{g} \mathrm{dm}^{-3}\right)$} & \multicolumn{2}{|c|}{ EE Naranjal } & \multicolumn{2}{|c|}{ EE Timbío } & \multicolumn{2}{|c|}{ FC Jamundí } \\
\hline $\mathrm{Ca}$ & $\mathrm{Mg}$ & $\mathrm{K}$ & Promedio & D.E. & Promedio & D.E. & Promedio & D.E. \\
\hline 0 & 0 & 0 & 1,54 & 0,40 & 1,01 & 0,03 & 0,88 & 0,18 \\
\hline 0 & 0 & 1 & 2,10 & 0,40 & 1,55 & 0,30 & 1,26 & 0,14 \\
\hline 0 & 0 & 3 & 2,80 & 0,47 & 2,65 & 0,16 & 1,90 & 0,57 \\
\hline 0 & 1 & 0 & 2,42 & 0,20 & 1,83 & 0,13 & 1,33 & 0,13 \\
\hline 0 & 1 & 1 & 3,31 & 0,34 & 2,46 & 0,12 & 1,80 & 0,24 \\
\hline 0 & 1 & 3 & 4,66 & 0,65 & 3,40 & 0,43 & 2,13 & 0,23 \\
\hline 0 & 3 & 0 & 5,13 & 1,34 & 3,48 & 0,39 & 2,40 & 0,33 \\
\hline 0 & 3 & 1 & 4,72 & 2,15 & 3,84 & 0,32 & 2,64 & 0,51 \\
\hline 0 & 3 & 3 & 5,47 & 1,26 & 4,78 & 0,62 & 3,51 & 0,41 \\
\hline 1 & 0 & 0 & 2,01 & 0,12 & 1,37 & 0,03 & 1,66 & 0,16 \\
\hline 1 & 0 & 1 & 2,77 & 0,86 & 2,17 & 0,14 & 1,92 & 0,16 \\
\hline 1 & 0 & 3 & 3,27 & 0,36 & 3,01 & 0,29 & 2,20 & 0,15 \\
\hline 1 & 1 & 0 & 2,83 & 0,13 & 2,08 & 0,21 & 2,11 & 0,13 \\
\hline 1 & 1 & 1 & 3,80 & 0,45 & 2,63 & 0,21 & 2,31 & 0,07 \\
\hline 1 & 1 & 3 & 5,10 & 0,37 & 3,14 & 0,53 & 2,79 & 0,32 \\
\hline 1 & 3 & 0 & 5,24 & 0,55 & 2,76 & 0,45 & 2,55 & 0,18 \\
\hline 1 & 3 & 1 & 5,41 & 0,62 & 3,95 & 0,50 & 2,55 & 0,10 \\
\hline 1 & 3 & 3 & 5,71 & 1,13 & 4,56 & 0,60 & 2,97 & 0,09 \\
\hline 3 & 0 & 0 & 2,07 & 0,10 & 1,60 & 0,04 & 1,70 & 0,14 \\
\hline 3 & 0 & 1 & 3,14 & 0,69 & 2,13 & 0,17 & 1,88 & 0,09 \\
\hline 3 & 0 & 3 & 4,03 & 0,29 & 3,30 & 0,25 & 2,19 & 0,10 \\
\hline 3 & 1 & 0 & 2,92 & 1,06 & 2,18 & 0,14 & 2,14 & 0,15 \\
\hline 3 & 1 & 1 & 3,82 & 0,25 & 2,85 & 0,27 & 2,36 & 0,28 \\
\hline 3 & 1 & 3 & 4,86 & 0,42 & 3,90 & 0,41 & 2,47 & 0,13 \\
\hline 3 & 3 & 0 & 5,03 & 0,05 & 3,49 & 0,29 & 2,73 & 0,18 \\
\hline 3 & 3 & 1 & 3,96 & 1,32 & 3,89 & 0,71 & 2,91 & 0,23 \\
\hline 3 & 3 & 3 & 6,25 & 0,85 & 5,39 & 0,85 & 3,17 & 0,17 \\
\hline
\end{tabular}




\section{Efecto de la salinidad en el crecimiento de la planta}

Conforme a los aumentos de la C.E. se presentó una disminución significativa del crecimiento de las plantas (Fig. 2). Si se asume una reducción del $10 \%$ en la biomasa de las plantas lo cual equivale al $90 \%$ de su materia seca total se obtienen los siguientes niveles críticos de la C.E. para los suelos de EE Naranjal, EE Timbío y FC Jamundí: 1,58 dS $\mathrm{m}^{-1}, 1,14 \mathrm{dS} \mathrm{m}^{-1}$ y $1,05 \mathrm{dS} \mathrm{m}^{-1}$, respectivamente. Estos valores son relativamente similares y reflejan las condiciones "naturales" del suelo, puesto que se aproximan mucho a los detectados en el tratamiento testigo. Con el fin de generar un solo modelo que pudiera expresar el efecto de la C.E. sobre el crecimiento de las plantas en las tres unidades de suelo, los valores absolutos de la materia seca total de las plantas fueron transformados en crecimiento relativo (Fig. 3). De acuerdo con el modelo seleccionado, y asumiendo el $90 \%$ del crecimiento relativo, se encontró un nivel crítico promedio de 1,1 dS $\mathrm{m}^{-1}$ para la C.E., el cual se aproxima más a los valores hallados para EE Timbío y FC Jamundí, que EE Naranjal.

Aunque en los resultados obtenidos no se descarta el efecto de la competencia entre los nutrientes suministrados, además de la influencia del ion sulfato, se considera que el factor determinante en el crecimiento de las plantas fue la salinidad.

Faria et al. (2009), Figueirêdo et al. (2006) y Karasawa et al. (2003) reportan valores críticos de C.E. similares a los calculados en este estudio $\left(1,2,1,2\right.$ y $0,9 \mathrm{dS} \mathrm{m}^{-1}$, respectivamente) para el crecimiento inicial de café en algunos suelos de Brasil (principalmente latosoles). A su vez,
Faria et al. (2009) observaron que la salinidad extrema resultante de la ferti-irrigación con urea y $\mathrm{KCl}$, causaba la muerte de las plantas. Lo expuesto difiere parcialmente de lo obtenido por Matiello (1999) para suelos con características similares, quien al aplicar agua salina a las plantas jóvenes de café mediante el sistema de goteo, observó cierta resistencia, desde que el agua no fuera excesivamente salina (hasta $\left.2,0 \mathrm{dS} \mathrm{m}^{-1}\right)$.

La alta salinidad resultante de la aplicación de las mayores dosis de fertilizantes en especial los sulfatos de $\mathrm{Mg}$ y $\mathrm{K}$, ocasionó la muerte de algunas plantas pocos días después del trasplante. En la unidad Chinchiná, el número de plantas muertas ascendió a 5, en Doscientos a 9 y en Timbío 13. En la Figura 4 se pueden observar las sintomatologías encontradas unos días después del trasplante.

En resumen se puede decir que, a excepción de pocas variaciones, en las tres unidades de suelo el comportamiento de la respuesta a los tratamientos fue similar, pues en todas las plantas el desarrollo se afectó negativamente conforme al incremento en las dosis de cualquiera de los tres nutrientes. La reducción promedia en el peso de las plantas fue mayor cuando se suministró $\mathrm{Mg}(69 \%)$, seguido por K $(40 \%)$ y Ca $(31 \%)$, efecto que se asoció principalmente a la salinidad generada por las fuentes fertilizantes; adicionalmente, al aumentar las dosis de Mg y K o con la aplicación simultánea de los dos elementos, las consecuencias negativas fueron aún mayores. 

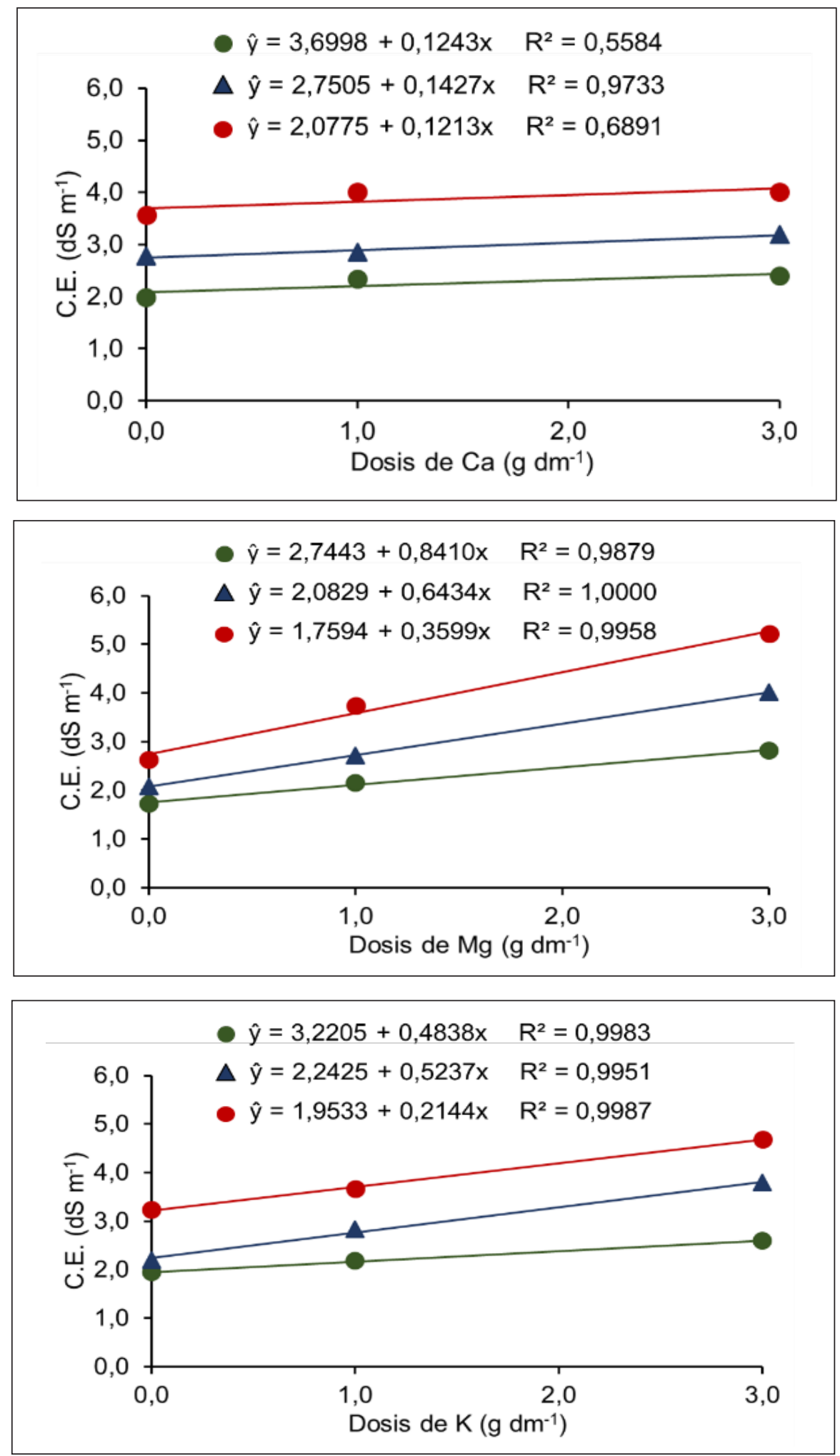

Figura 1. Conductividad eléctrica de tres suelos cafeteros en respuesta a dosis de $\mathrm{Ca}, \mathrm{Mg}$ y K 

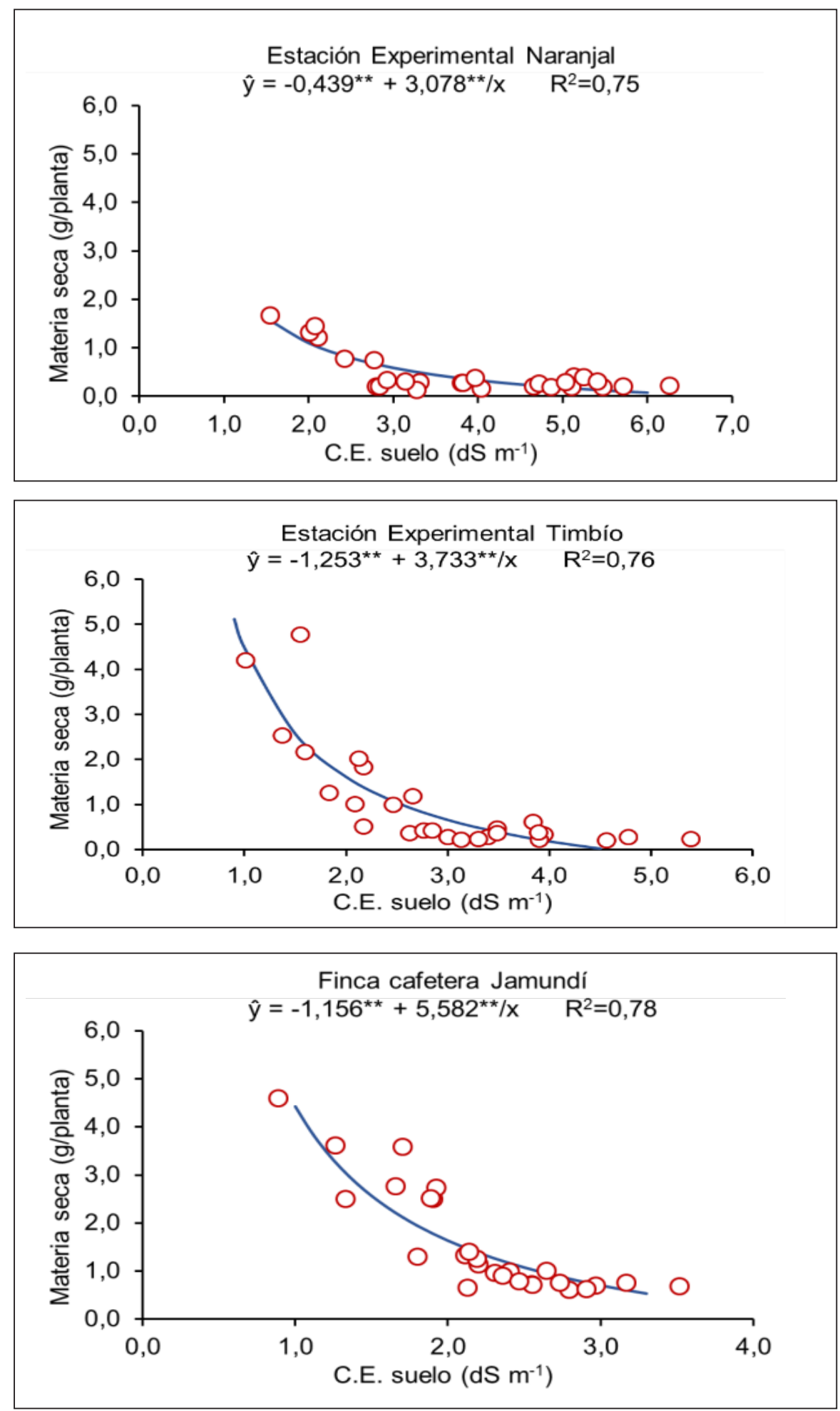

Figura 2. Materia seca de plantas de café durante la etapa de almácigo en respuesta a la conductividad eléctrica (C.E.) en tres suelos de la zona cafetera 


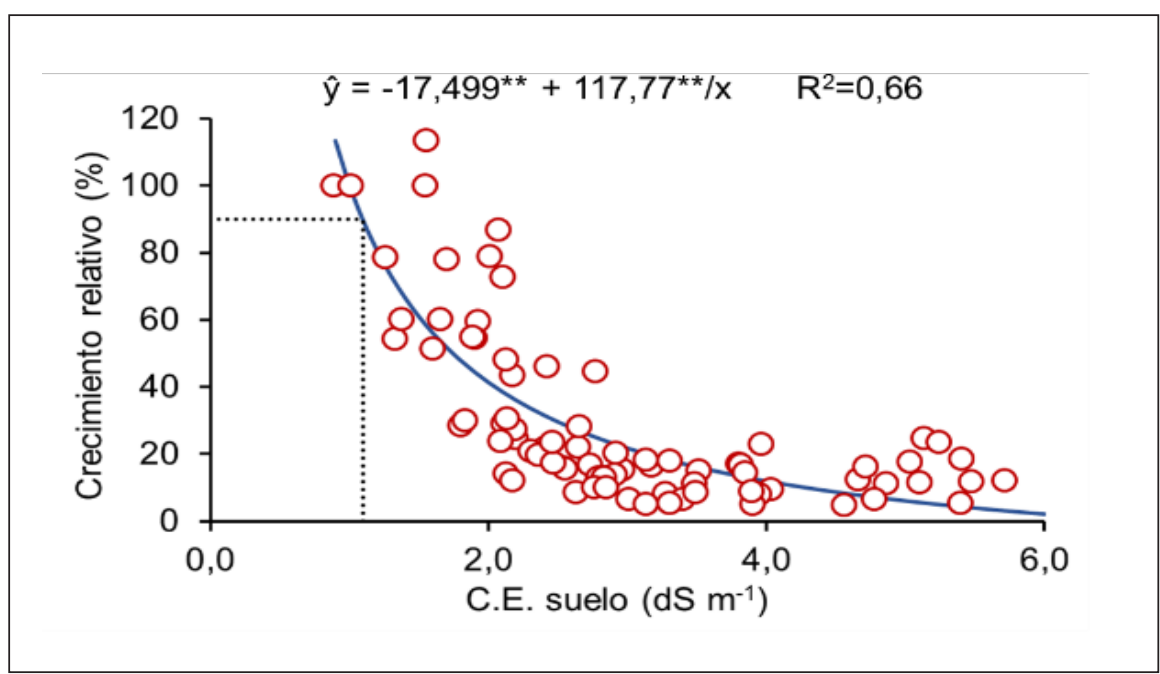

Figura 3. Crecimiento relativo de plantas de café durante la etapa de almácigo en función de la conductividad eléctrica del suelo (C.E.)(n=81)

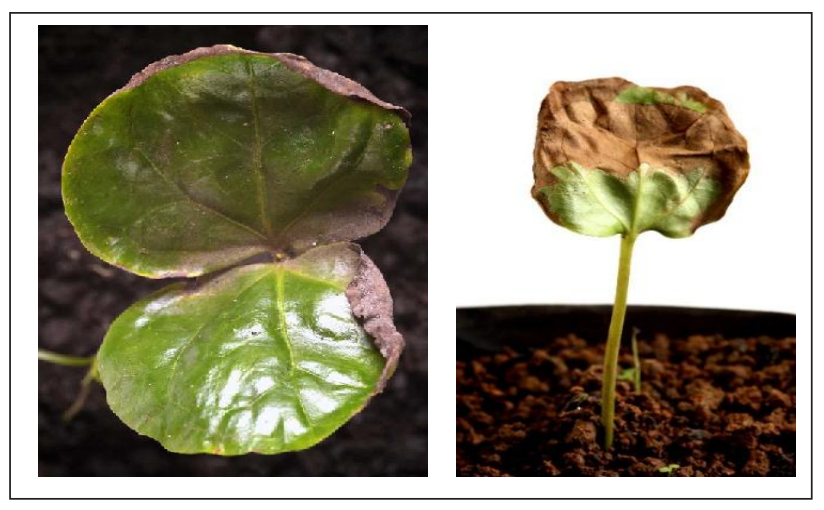

Figura 4. Síntomas de toxicidad por salinidad, causada por las dosis más altas de sulfatos de magnesio y potasio $\left(3 \mathrm{~g} \mathrm{dm}^{-3}\right)$, en los suelos de la EE Naranjal (izquierda) y la FC Jamundí (derecha), cinco días después del trasplante

\section{CONCLUSIONES}

La salinidad del suelo, generada por la aplicación de fertilizantes, depende tanto de la cantidad y la fuente empleada, como de las propiedades del suelo.

El café tolera niveles bajos de salinidad durante la fase de almácigo (conductividades eléctricasC.E. menores a $1,1 \mathrm{dS} \mathrm{m}^{-1}$ ).

\section{BIBLIOGRAFÍA}

AMÉZQUITA, C. E. 2001. Las propiedades físicas y el manejo productivo de los suelos. En: Silva F. (ed.). Fertilidad de Suelos, diagnóstico y control. 2a. edición. Sociedad Colombiana de la Ciencia del Suelo. 137 - 154 p.

ÁVILA R., W.E., SADEGHIAN K., S., SÁNCHEZ A., P.M. y CASTRO F., H.E. 2010. Respuesta del café al fósforo y abonos orgánicos en la etapa de almácigo. Centro de Investigaciones del Café. 61(4):358 - 369.

CRAMER, G.R., ALBERICO, G.J. y SCHMIDT, C. 1994. Salt tolerance is not associated with thesodium accumulation of two maize hybrids. Australian Journal of Plant Physiologic. 21:675 $-692$.

FARIA, F.E.S., LIMA, L.A., REBEIRO, M.S., REZENDE F.C. y CARVALHO J.G. 2009. Efeito de parcelamento da fertirrigação com N e K e salinidade do solo no crescimento inicial de cultivares de cafeeiro. Irriga.14(2):145 - 157. 
FIGUEIRÊDO, V. B., FARIA, M. A. y SILVA, E. L. 2006. Crescimento inicial do cafeeiro irrigado comágua salina e salinização do solo. Revista Brasileira de Engenharia Agrícola e Ambiental. 10(1):50 - 57.

GARCÍA, O. A. 2010. Manejo de la degradación de suelo. En: Burbano, H. y Siva, F., eds. Ciencia del Suelo. Principios básicos. Bogotá: Sociedad Colombiana de la Ciencia del Suelo. 309 - 356 p.

GUERRERO R., R. 2004. Manual Técnico. Propiedades generales de los fertilizantes sólidos. Monómeros Colombo-Venezolanos, Bogotá. 46 p.

INSTITUTO COLOMBIANO AGROPECUARIO (ICA). 1992. Fertilización en diversos cultivos, Quinta aproximación. P 64. En: Manual de Asistencia Técnica No. 25. Centro de Investigaciones Tabaitatá, Bogotá.

KARASAWA, S., EGUCHI, E. S., MIRANDA, J. H., DUARTE, S. N.y KARASAWA, M. M. G. 2003. Desenvolvimento de mudas de cafeeiro (Coffea arabica L.) irrigadas com água salina. Engenharia Rural. 14:1 - 12.

MATIELLO, J.B. 1999. Evolução da salinidade e comportamento do cafeeiro em área irrigada por gotejamento em Brejões, BA. p.185. In: Congresso Brasileiro de Pesquisa Cafeeira, 25 Franca.Anais. Franca: CP\&D. p.185.

NAZÁRIO, A.A., GARCIA. G.O., GONÇALVES, I.Z, MADALÃO, J.C. y ARAUJO, G.L. 2010. Crescimento do cafeeiro conilon irrigado com água salina. Engenharia Ambiental-Espírito Santo do Pinhal. 7(3):178 - 195.

MARSCHNER, H. 1995. Mineral nutrition of higher plants. Second edition. Academic Press. $889 \mathrm{p}$.
LINDSAY, W.L. 2001. Chemical equilibria in soils. Ney Jersey, Blackburn. 449 p.

RICHARDS, L.A. 1954. Diagnosis and Improvement of Saline and Alkali Soils. Washington, Unite States Department of Agriculture. Agriculture Handbook. 60:160 p

SALAMANCA, J., A. y SADEGHIAN K., S. 2008. Almácigos de café con distintas proporciones de lombrinaza en suelos con diferente contenido de materia orgánica. Centro de Investigaciones del Café. 59(2):91 - 102.

SADEGHIAN, K., S. 2013. Nutrición de cafetales. p. 85-116. En: CENICAFE. Manual del cafetero colombiano: Investigación y tecnología para la sostenibilidad de la caficultura. Centro de Investigaciones del Café. Chinchiná. 